Musées, Patrimoine et Culture scientifiques et techniques

$129 \mid 2010$

mai - juin 2010

Importance des collections de Sciences naturelles pour la connaissance de la biodiversité

The importance of Natural Science Collections for biodiversity knowledge

Alain Canard, Jean-Claude Beaucournu, Joël Boustie et Dominique Bernard

(2) OpenEdition

Journals

Édition électronique

URL : http://journals.openedition.org/ocim/167

DOI : 10.4000/ocim. 167

ISSN : 2108-646X

Éditeur

OCIM

Édition imprimée

Date de publication : 1 mai 2010

Pagination : $36-40$

ISSN : 0994-1908

Référence électronique

Alain Canard, Jean-Claude Beaucournu, Joël Boustie et Dominique Bernard, «Importance des collections de Sciences naturelles pour la connaissance de la biodiversité ", La Lettre de I'OCIM [En ligne], 129 | 2010, mis en ligne le 01 mai 2012, consulté le 30 avril 2019. URL : http:// journals.openedition.org/ocim/167; DOI : 10.4000/ocim.167 


\title{
Importance des collections de Sciences naturelles pour la connaissance de la biodiversité
}

\author{
Alain Canard, Jean-Claude Beaucournu, \\ Joël Boustie et Dominique Bernard *
}

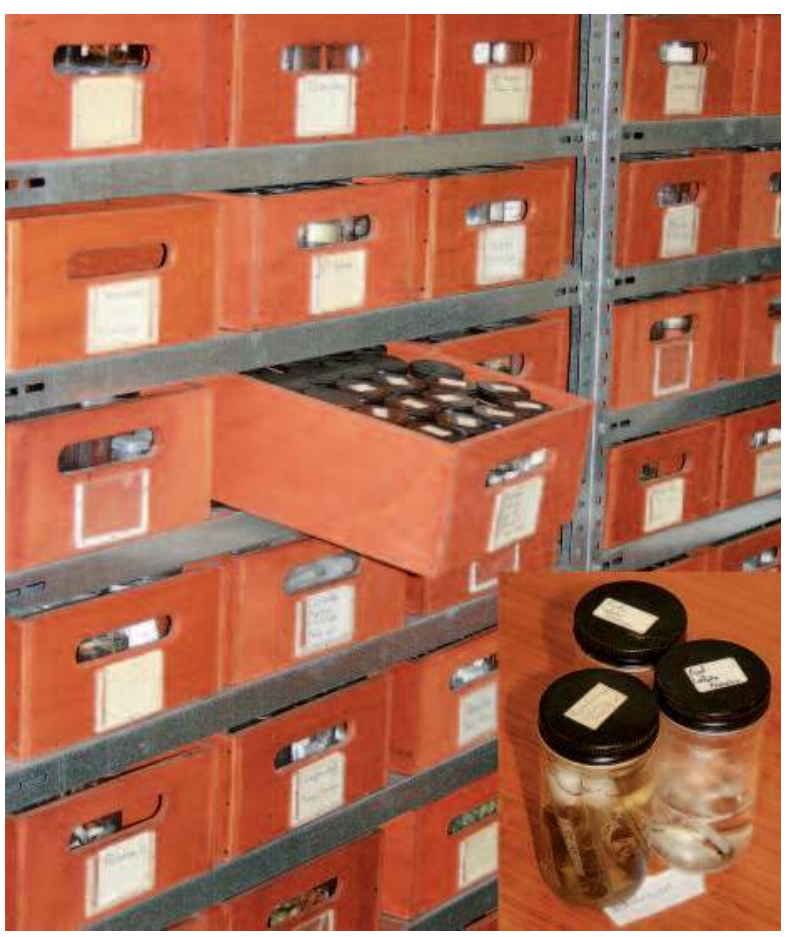

La collection d'araignées de l'université de Rennes 1 : meubles, flacons et tubes (c) Université de Rennes 1

* Alain Canard est directeur de l'URU420 Biodiversité fonctionnelle et gestion des territoires et responsable du projet Arche des sciences Jean-Claude Beaucournu est ancien directeur du laboratoire de Parasitologie Joël Boustie est directeur de l'unité de recherche en Sciences pharmaceutiques Substances lichéniques et photoprotection Dominique Bernard est vice-président de la commission de Culture scientifique et technique
Bien traitées puis délaissées, les collections de spécimens constituent maintenant une ressource indispensable pour les études sur la biodiversité et ses évolutions : l'exemple de l'université de Rennes 1 montre que les collections devraient retrouver un statut en rapport avec leur intérêt mais qu'un problème reste à régler, celui de la disparition des chercheurs travaillant dans le secteur de la systématique.

Les collections de Sciences naturelles ont été considérées comme indispensables au cours des derniers siècles mais depuis une cinquantaine d'années, elles étaient négligées, d'une part car l'on pensait que les espèces étaient bien connues et d'autre part car l'on n'envisageait pas la disparition de la biodiversité. Les techniques nouvelles d'identification nous font reconsidérer le nombre des espèces dont les descriptions, réalisées souvent à partir de spécimens en collection, sont en augmentation très rapide. La faible connaissance sur la distribution du plus grand nombre des espèces redonne une valeur fondamentale aux spécimens mis en collection.

\section{Les collections}

Les collections concernant la biodiversité actuelle sont issues de récoltes de matériel biologique effectuées sur le terrain, référencées en ce qui concerne les dates et les sites de captures. Il s'agit parfois de prélèvements 
d'animaux ou de végétaux dont les espèces n’ont pas été encore identifiées et qui ont été mis collectivement dans un conteneur destiné à chaque prélèvement. Dans certains cas, les spécimens identifiés jusqu'à l'espèce, sont conservés séparément. Il peut s'agir aussi de prélèvements de tissus animaux, donc d'organismes incomplets mais dont le génome contenu dans les cellules est suffisant pour rendre compte à la fois de l'individu dans sa population et de l'espèce parmi les autres. Certaines de ces collections ont été réalisées il y a fort longtemps, les spécialistes référents font maintenant défaut et leur utilisation à des fins de recherche est devenue exceptionnelle. D'autres servent encore de support à des recherches, notamment sur la Biodiversité, ce qui leur permet d'être parfois qualifiées de «collections vivantes».

\section{Le rôle traditionnel des collections en recherche sur la biodiversité}

Un principe qui devrait obligatoirement être respecté, veut que les noms d'espèces attribués à des spécimens pour des travaux de systématique soient corrects et correspondent bien aux espèces décrites comme telles. En Botanique, ou en Zoologie, lorsqu'une espèce est décrite, le ou les spécimens ayant servi à cette description sont déposés dans un musée et sont nommés «types ». Un type ou une série type est unique, c'est le support concret de la description de l'espèce réalisée dans un ouvrage scientifique et effectuée parallèlement à son dépôt. Les types servent de modèle de référence pour tous les individus trouvés ultérieurement que l'on pourra assimiler à la même espèce. Mais, pour des raisons pratiques, il n'est pas possible de se référer pour chaque identification de spécimens à des types. Les ouvrages d'identification permettent en général de reconnaître séparément les espèces, mais en cas de doute, il est plus prudent de se référer à des spécimens de collection. Les collections de référence sont vérifiées, parfois à partir des types mais le plus souvent, indirectement par des spécialistes du groupe. Il est avéré que l’incertitude sur les identifications est d'autant moindre qu'elle est basée à la fois sur l'emploi des ouvrages et la confrontation à des spécimens des collections de référence. En effet, contrairement à une idée reçue, les collections servent dans les identifications d'un bon nombre d'organismes vivants et, si on les utilise peu pour identifier les animaux vertébrés, elles sont très employées pour les végétaux et les invertébrés (regroupement artificiel d'espèces) soit pour plus de $96 \%$ de presque deux millions d'espèces vivantes répertoriées. Les identifications de vertébrés ne requièrent pas les mêmes précautions car elles s'adressent à beaucoup moins d'espèces et s'opposent à un souci évident de respect des populations. Il n'est heureusement pas indispensable de capturer tous les oiseaux pour les identifier, ni de les tuer et de les conserver pour se garantir d'erreurs d'identification ou bien encore déceler de nouvelles espèces. Si pour des régions géographiques bien connues, comme la nôtre, il existe quelques contestations de spécialistes pour des identifications inhabituelles, elles ne justifient pas de mises en collection systématique des spécimens.

\section{L'importance nouvelle des collections, données générales}

\section{La systématique et les descriptions d'espèces}

$\mathrm{Si}$, pendant plusieurs décades, que l'on pourrait situer entre 1960 et actuellement, les musées ont très peu mis en collection de spécimens, hors les types, cet état de fait est actuellement un handicap pour les études sur la biodiversité. En effet, les identifications étaient limitées aux caractères morpho-anatomiques des spécimens mais le développement de techniques nouvelles telles la cladistique et la génétique moléculaire permettent maintenant de caractériser et d'identifier des espèces soit sur une partie de leur génome soit par des analyses multivariées. Une conséquence de ces nouvelles techniques est que le nombre des espèces connues qui évoluait lentement au milieu du XXe siècle, s'est mis à croître rapidement. Les nouvelles espèces décrites proviennent parfois du terrain mais aussi, pour beaucoup, de spécimens conservés en collections. Elles sont découvertes soit dans des prélèvements non encore identifiés, soit aussi parmi des spécimens attribués à une espèce proche. Il est évident que l'absence de mise en collection pendant un demi-siècle limite, du fait de cette carence, la découverte de nouvelles espèces.

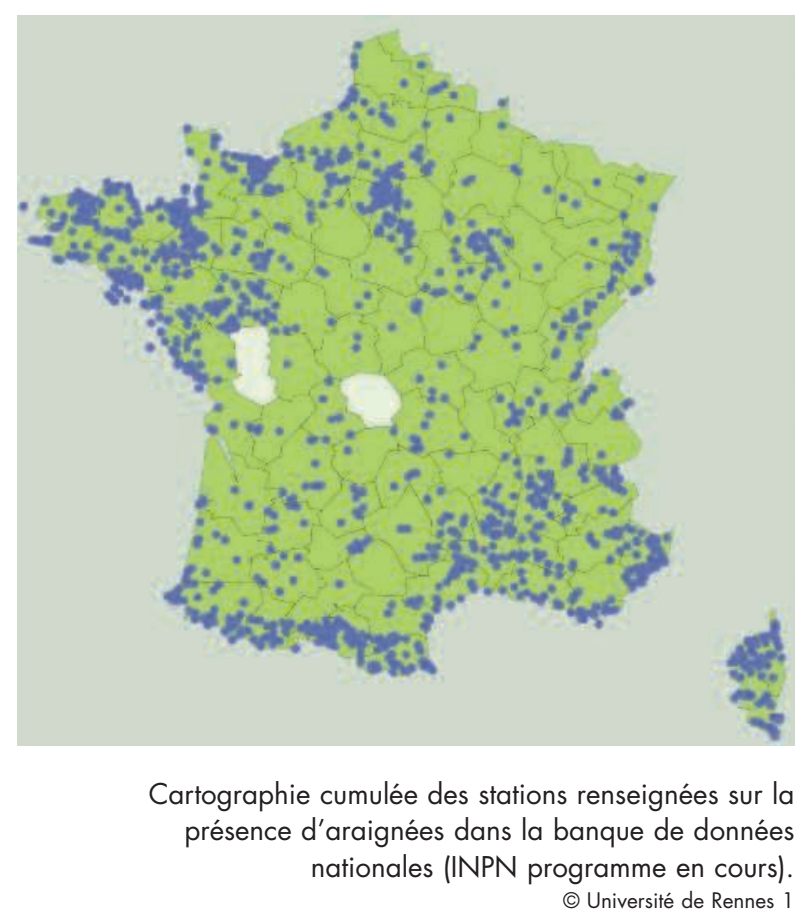


La connaissance de la biodiversité et de son évolution

Les distributions géographiques des espèces avaient semble-t-il jusqu'ici une certaine stabilité ; aussi l'attention portée à leur présence se relâchait nettement. Les voyages et les échanges internationaux s'accélérant, de très nombreux individus vivants ont été introduits, accidentellement ou non, provoquant des déséquilibres ponctuels ou durables dans les nouveaux milieux où ils réussissent à se développer. Ces espèces ont parfois été introduites au cours du dernier demi-siècle sans que l'on ait relevé leur présence. Elles se sont développées d'abord lentement et deviennent maintenant envahissantes en faisant courir des risques importants aux équilibres locaux. Les conséquences néfastes des ces invasions étant aussi parfois économiques, lintérêt porté alors à la biodiversité en est d'autant plus important. Il aurait été intéressant de connaître l'histoire de ces colonisations pour mieux prévoir les invasions futures et imaginer des actions de protection mais les éléments objectifs manquent, en l'occurrence l'absence de données sur les présences antérieures.

Les changements climatiques globaux paraissent actuellement évidents mais ces changements étaient moins nets il y a quelques décades. On voit par contre des modifications de plus en plus importantes dans la distribution de certaines espèces. Là encore, des prélèvements mis en collection permettent de mieux cerner la colonisation de celles-ci en corrélation avec les changements climatiques.

Les publications de listes d'espèces qui étaient nombreuses jusque vers 1980 ont disparu progressivement car, acceptées et publiées presque toujours dans des revues « régionales ou nationales ». Leurs facteurs d'impacts sont inexistants ou faibles. N'étant plus prises en compte dans l'avancement ou les évaluations des chercheurs et des laboratoires, les scientifiques dédaignent de publier ce type de travaux.

On constate actuellement des modifications récentes et rapides dans les distributions géographiques de certaines espèces, bien qu'il existe une forte diminution des références publiées. Les principales données disponibles, et vérifiables sont donc celles disponibles à partir des collections.

\section{L'intérêt des collections à travers trois exemples choisis parmi les collections de l'université de Rennes 1}

\section{Les collections de puces}

Les puces sont des vecteurs de maladies importants, tant pour l'homme que pour d'autres animaux mais c'est plutôt dans un objectif de recherche fondamentale qu'un médecin de l'université de Rennes 1, Jean-Claude

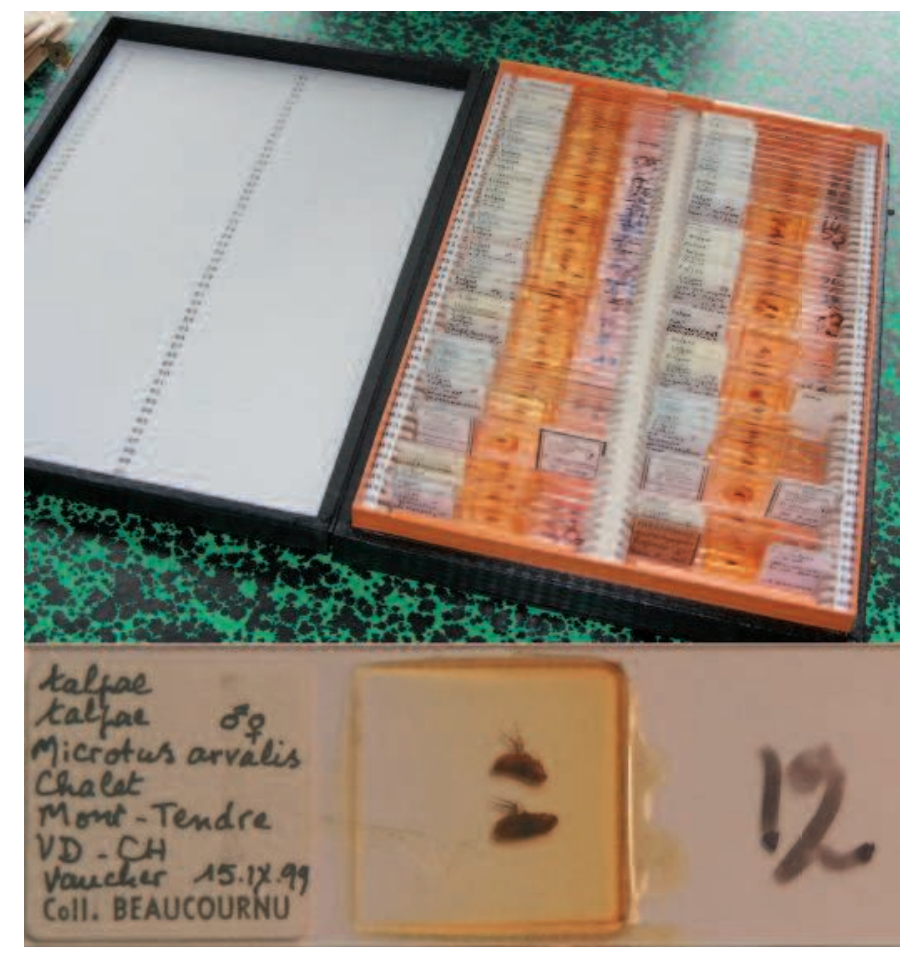

Boîtes et lames contenant une partie de la collections de puces. (c) Université de Rennes 1

Beaucournu, a étudié ce groupe. Sur les 2700 espèces mondiales, environ 250 ont été décrites, ou redécrites à Rennes et les types appartiennent, pour la plupart, à la collection de Rennes. Toutes les puces de la faune de France y sont représentées, mais aussi de très nombreuses autres, répandues du Sptizberg à l'Antarctique! Les taxa identifiés et présents dans cette "Siphonaptèrothèque » (environ 950) servent de référence pour des identifications nouvelles par des chercheurs étrangers. La valeur de cette collection impose une maintenance considérable car les demandes pour vérification sont nombreuses.

De ce point de vue, cette collection est encore « vivante » et l'université considère encore quili est de son devoir de la conserver en bon état. Un effort est effectué actuellement pour en informatiser le contenu et le porter à la connaissance des chercheurs, afin quà Rennes, ou ailleurs, la collectivité mondiale des chercheurs puisse l'exploiter. Relevons d'ailleurs que c'est grâce à la consultation d'importantes collections étrangères ("Collection Rothschild » du Natural History Museum de Londres, ou de la Smithsonian Institution à Washington) que le statut de nombreux taxons a pu être révisé, ceci permettant, la plupart du temps, d'apporter de nouvelles « entités » à l'ordre des Siphonaptères. Enfin, parallèlement, le nombre d'espèces de Rickettsies ou d'autres bactéries pathogènes, voire de virus, découverts chez les puces ne cesse de croître. 


\section{Les collections d'araignées}

Les collections d'araignées sont récentes et ont été constituées dans les 30 dernières années. Il n'y a pas de types, mais des séries de prélèvements sur tout le territoire national. Elles recouvrent un millier d'espèces sur les 1600 environ présentes en France métropolitaine. L'intérêt de ces collections tient d'une part dans les services qu'elles rendent lors des identifications de spécimens nouveaux qu'ils soient découverts sur le terrain ou dans les spécimens conservés, et d'autre part, dans les données qu'elles apportent sur la connaissance de la biodiversité et de son évolution. Actuellement, les collections servent pour des identifications dans le but de tester la diversité de certains milieux naturels La liste des espèces présentes et leur distribution sont informatisées dans une banque générale. Elles servent pour comparer l'échantillon réalisé dans un biotope avec la communauté d'araignées que l'on peut s'attendre à trouver dans le même type de biotope, dans la même région biogéographique. Des indices sont calculés à partir de cette comparaison des communautés d'araignées et permettent d'évaluer, à travers elles, la richesse et l'originalité des milieux étendues à une grande partie de la faune (macroinvertébrés épigés). Ce conglomérat de groupes d'animaux représente très souvent dans les milieux naturels des densités de plus d'un millier d'individus par mètre carré. Les spécimens tués et conservés pour constituer des collections deviennent donc ainsi des supports de connaissance pour des mesures de protection des biotopes et des araignées elles-mêmes. Les données de distribution servent aussi à suivre l'évolution des colonisations des milieux par des espèces. Dans les collections de Rennes 1, huit espèces nouvellement décrites ont été identifiées alors qu'elles avaient été confondues avec d'autres. Ces identifications, bien que tardives, ont permis de mieux connaître la distribution de ces espèces nouvelles.

Malgré les efforts actuels, les banques de données sont encore assez peu fournies, et un programme national est en cours pour améliorer cet état.

\section{Les collections de lichens}

Henry Nicollon des Abbayes, professeur à la faculté des Sciences de Rennes, réalise des collections de botanique entre 1947 et 1971. Lichénologue de renom, il est l'auteur de La Flore et la végétation du Massif Armoricain - Flore vasculaire en 1971. Entre les années 1930 et 1960, il établit une remarquable collection de lichens et décrit de nombreuses espèces dont plus de 90 types. À son départ de l'université, malgré l'absence de programme général de conservation, des enseignants-chercheurs ont assuré des échanges et conservé ces collections en dehors de leurs tâches principales. Au début des années 2000, une équipe de recherche en Pharmacie qui isole et identifie des molécules originales bioactives a choisi d'étudier les métabolites secondaires produits par les lichens. Sans l'aide de l'enseignant-chercheur retraité qui a préservé et transmis cette collection, ce type de recherche qui s'affirme aujourd'hui, n'aurait pu être initié. Il ne s'agit pas d'utiliser cette précieuse collection pour des travaux phytochimiques mais c'est une base de référence pour une bonne identification des espèces en amont, ce qui est absolument fondamental. La qualité de la collection permet les comparaisons et facilite l'identification des espèces collectées pour rechercher de nouveaux médicaments ou des molécules d'intérêt. Les informations de localités et d'écologie contenues dans la collection sont aussi très précieuses pour connaître les sites propices à la collecte d'un lichen particulier.

Une collection « vivante » à l'époque de Nicollon des Abbayes, employée activement lors de recherches en

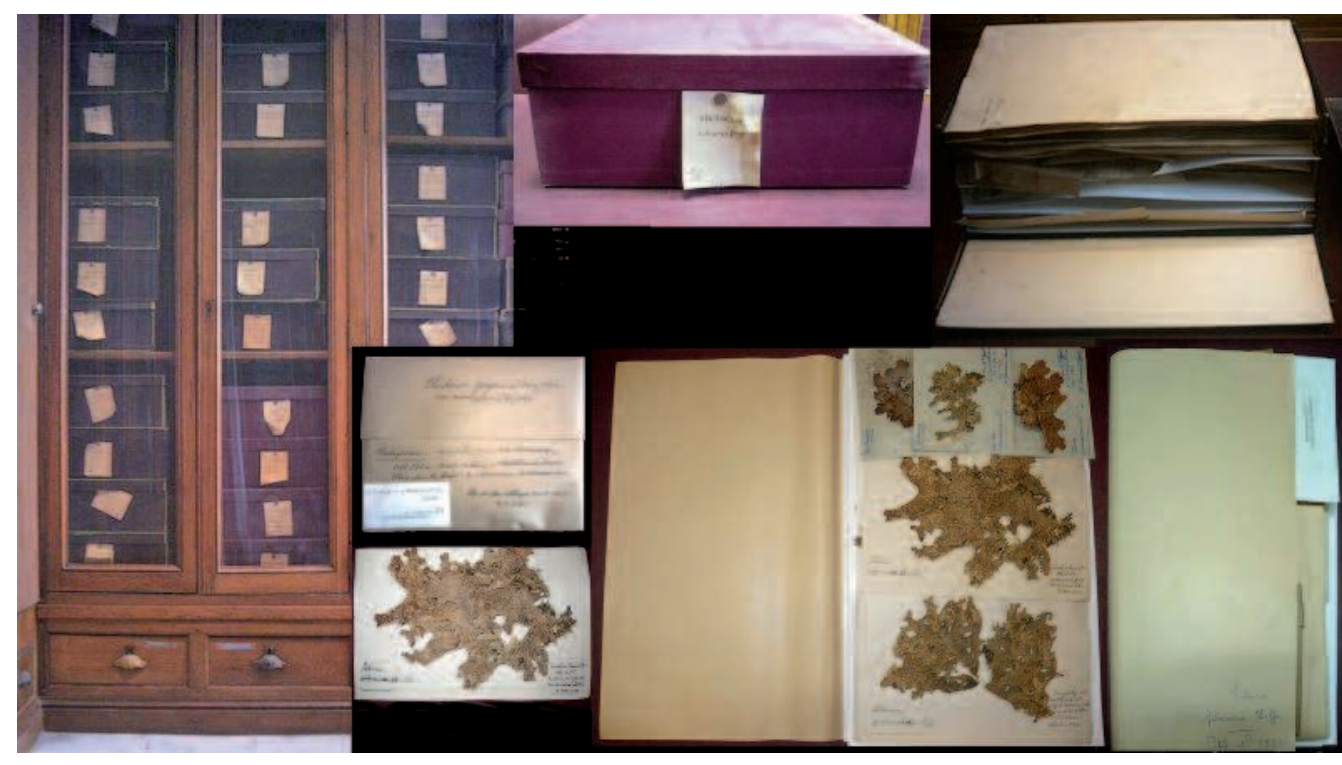

Quelques spécimens de la collection de lichens (๑) Université de Rennes 1 
systématique peut sommeiller pendant quelques années, puis retrouver ainsi une « nouvelle vie » très active quelques années plus tard.

\section{Conclusion}

Les collections d'animaux et de végétaux ont été considérées comme issues d'un système de recherche dépassé. Non seulement il n'en est rien mais, de plus, les collections de matériel biologique sont à la source de nouvelles recherches en systématique et en biodiversité. Les biologistes avaient d'autant plus tendance à ne pas les conserver qu'une idée s'était développée supposant que la mise en collection nuisait par les prélèvements de faune à la préservation des espèces. Ce point est discutable si l'on met en parallèle les destructions d'espèces vivantes par la mise en collection avec celles imposées par les destructions d'habitats dans les activités humaines quotidiennes. Que représentent les 60000 spécimens de la collection d'araignées de l'université de Rennes 1 sachant qu'un mètre carré d'espace naturel contient plusieurs dizaines à plus d'un millier d'araignées, surtout si l'on met en relation les milliers d'hectares ( 1 ha $=10000 \mathrm{~m}^{2}$ et doit abriter plusieurs millions d'araignées) modifiés ou détruits chaque année par les constructions humaines, habitations, routes... Ceci sans tenir compte des autres destructions par pesticides ou pollutions.

De nouvelles possibilités en recherche permettent, à l'aide de techniques de génétique moléculaire, d’estimer les distances génétiques entre individus et par là d'étudier les populations, autre dimension de la biodiversité. Des spécimens entiers ne sont pas indispensables pour ces identifications, aussi le dépôt de prélèvement de tissus peut suffire. Le développement de ces nouvelles techniques permet de distinguer des espèces les unes des autres en raison de différences dans leurs barecode moléculaire (séquence d'ADN identifiée et répertoriée) ceci avec une rapidité de plus en plus grande. Le programme "barecoding of life » vise à augmenter le séquençage des gènes des espèces et à augmenter le nombre de barecodes d'espèces connues. Il est employé seul pour distinguer des espèces ou supposées telles lorsque ces espèces sont inconnues, mais il ne dispense pas d'une mise en collection des spécimens, référence tangible de leur présence.

Identifier les espèces nécessite des connaissances approfondies sur les caractères d'identification qui sont multiples. Cela nécessite parfois un travail long et fastidieux qui est ignoré à la fois de certains milieux scientifiques et de nombreux organismes. De plus, ces recherches longues ne permettent pas la valorisation des résultats par des publications dans des revues à facteur d'impact élevé. Seuls les articles référencés dans ces conditions étant pris en compte, les chercheurs travaillant dans ce secteur ne sont pas considérés comme performants. On constate en conséquence une diminution du nombre des chercheurs travaillant en systématique et parallèlement une baisse de la mise en collection de spécimens. Les départs à la retraite de chercheurs dans le domaine de la systématique ne sont pas compensés par des arrivées de jeunes chercheurs dans le même domaine. Cela est particulièrement grave car les sciences naturalistes sont un domaine où la transmission du savoir est un facteur clé. À l'échelle d'une vie, il n'est guère possible de progresser s’il faut redécouvrir par soi-même toutes les données qui ne sont que très médiocrement décrites dans les ouvrages. Il en résulte que des groupes systématiques importants n'ont plus de spécialistes, ce qui pose un problème actuel pour la connaissance de la biodiversité.

Les collections de Sciences naturelles constituent un patrimoine dont la valeur augmente encore du fait de la protection des espèces. Les spécimens des espèces protégées ne pouvant plus être récoltés, la valeur patrimoniale de ceux actuellement en collection en est d'autant plus importante.

Malgré leur coût de maintenance élevé en moyens financiers et en personnel, les collections d'espèces animales et végétales ont un rôle qui a considérablement augmenté en intérêt dans les recherches actuelles, y compris dans celles qui visent à conserver la biodiversité. Mais, même si l'on suppose que ces problèmes de moyens puissent se régler, le problème de la recherche sera lui plus difficile à résoudre. Il faudra une durée assez longue pour que de nouveaux chercheurs s'investissent dans les domaines abandonnés, bien qu'indispensables, et surtout qu'ils y trouvent un intérêt.

\section{Bibliographie}

Beaucournu, J.-C. et Launay, H. Siphonaptères de France et de Méditerranée occidentale, Faune de France, n76, 1990, 548 p.

Boustie, J. et Grube, M. Lichens, promising sources of bioactive secondary metabolites, Plant Genetic Resources : Characterization \& Utilization, 2005, 3(2), pp. 273-287.

Canard, A. Catalogue of Spiders Species from Europe and the Mediterranean basin, Revue Arachnologique, 2005 (part 1 et 2), pp. 1$255 ; 2008$ (part. 3 et 4), pp. 1-94.

De Graves, S., Cai, Y. et Anker, A. Global diversity of shrimps (Crustacea : Decapoda : Caridea) in freswater, Hydrobiologia, n595, 2008, pp. 287 293. 